\title{
Correlation Energy in the Unfamiliar Realm of Capacitors and Batteries
}

Non-mean-field theory of anomalously large double-layer capacitance. M. S. Loth, Brian Skinner, B. I. Shklovskii, Phys. Rev. E 82, 016107 (2010); arXiv: arXiv:1005.1693

A model of large volumetric capacitance in graphene supercapacitors based on ion clustering

Brian Skinner, M. M. Fogler, B. I. Shklovskii

arXiv:1109.0978

\section{Recommended and a Commentary by Chandra Varma, University of California, Riverside}

The correlation energy, defined as the correction to the mean-field or HartreeFock energy entered physics through Wigner's discussion of the low density electron gas. Considerable progress in understanding correlation energy in Jellium was achieved analytically and numerically but investigations of correlations in a periodic lattice became less fashionable due partly to the immense mathematical difficulties of the problem and partly due to the unreasonable success of the density functional theory in many of the properties of metals and semiconductors. This type of less than systematic mean-field theory itself failed for transition metal oxides which are Mott Insulators and for the normal state properties of the high temperature superconducting materials. In the last twenty-five years the intense effort to understand these and associated problems has led to interesting, new, and sometimes wayward direction of investigations of the physics due to correlations.

Now we have a new development inspired by experimental results in a completely different context, electrochemical capacitors and batteries. These are problems in classical statistical electro-physics which have remained untouched by theory for almost 100 years. Experiments reveal that mean-field DebyeHuckel type theories of screening (called Guoy-Chapman theories in this context) applied to interfaces between metals and insulators or electrolytes in contact with metals often fail spectacularly. For example in the latter the low frequency properties, due to the finite conductivity of the electrolyte, are dominated by an electrical double layer near the interface. If the measured double layer capacitance is parametrized by $C=\epsilon S /(4 \pi d)$, where $\epsilon$ is the independent measured dielectric constant of the insulator or electrolyte and $S$ is the surface area, $d$ comes out significantly less than an atomic radius (the Helmholtz limit) in many cases, leave alone a screening radius. This means that the traditional theory is inapplicable.

Consider two metal electrodes in an electrolyte, solid or liquid, in which the conductivity is such that apart from near the interfaces with the metal, charge 
neutrality is maintained. In the first paper highlighted above, the authors have considered some of the various contributions to the free-energy of the problem with the assumption that the mobile ions of the electrolyte are discrete objects, not a continuum as in the old theories, and including the correlation energy of the ions near the surfaces approximately. Three principal contributions to the energy are (1) the image charge attraction of the electrolyte-ions with the metal or the chemical interaction energy of these ions with the metal. Suppose this is very large compared to the temperature. Then a depletion layer is formed in the electrolyte even without the application of an external voltage. (2) At equilibrium the depletion energy of the electrolyte is about the same as the former. (3) A dipole layer under these conditions is formed at the interfaces by the charges in the electrolyte and the corresponding image charges or correlated ions in the metal. These dipoles are oriented in the residual electric field of the two former contributions. The dipole-dipole interaction energies are then repulsive, comparable to the ambient temperature. So a correlated nearly two-dimensional liquid of the ions plus associated neutralizing entities in the metal is formed. When a small voltage is applied across the electrodes, the charge distribution on the electrolyte changes negligibly because of the large binding energy; the charge induced on the metal plates by the voltage is accompanied by the change in the dipole-dipole energy. Then the capacitance $\mathrm{C}$ can be very large because $\mathrm{C}$ is the net compressibility of charge in the system, $C \approx e^{2}\left(\partial^{2} E_{d d} / \partial n^{2}\right)^{-1}$, where $\Delta E_{d d}$ is the small change in the sum of the dipole energy at the two interfaces for a given change in charge $\Delta n$ to the outside due to the voltage applied. (The phenomena may be compared, not quite accurately since the electrolyte problem is classical, to the large energy of the Mott gap in transition metal oxides leaving a residual effect, the formation of localized spins and their mutual interactions which dominates the low energy thermodynamics and magnetic response. Only, when the voltage applied is comparable to the energy of un-binding of the ions, the larger energy comes to play and the differential capacitance drops precipitously to Guoy-Chapman type values. Remarkably, experiments show trends similar to this.

These ideas have been extended to an electron gas between two electrodes and to another experimental system of great interest: capacitors formed by graphite electrodes, which display enormous capacitances and may in fact become useful for energy storage. In this case, the ions intercalate in graphite, forms clusters. The elastic energy of interactions of such clusters does what dipolar interactions did for the first case.

Considerable interest has developed recently in quantum-correlation problems at controlled interfaces of different solids, read Andrew Millis in September 2011 issue of the Journal Club. It would appear that there is also a whole class of classical problems, where because the interaction energies are at various scales, from much larger than the entropic energies to those comparable, for example macromolecules in solutions, where considerations similar to those considered in the papers above and their refinements play an important role. 